Revue internationale P.M.E.

Économie et gestion de la petite et moyenne entreprise

Revue

internationale

PME

\title{
La perception du succès des alliances stratégiques
}

\section{Marc Ingham}

Volume 4, numéro 2, 1991

URI : https://id.erudit.org/iderudit/1008063ar

DOI : https://doi.org/10.7202/1008063ar

Aller au sommaire du numéro

Éditeur(s)

Presses de l’Université du Québec

ISSN

0776-5436 (imprimé)

1918-9699 (numérique)

Découvrir la revue

Citer cet article

Ingham, M. (1991). La perception du succès des alliances stratégiques. Revue internationale P.M.E., 4(2), 43-83. https://doi.org/10.7202/1008063ar

\section{Résumé de l'article}

Cet article présente les résultats d'une enquête-pilote sur les alliances stratégiques. Il s'intéresse en particulier à la perception qu'ont les responsables d'entreprises du succès des accords. Il tente de relever les différences d'opinion chez les dirigeants de PME ou de plus grandes entreprises, ou selon que les personnes interrogées appartiennent à une entreprise ayant ou non une expérience en matière d'alliances. Les propositions soumises aux répondants sont issues de la littérature récente. Dans la première partie de l'article, nous présentons la perception qu'ont les responsables d'entreprises des fonctions sur lesquelles portent en priorité les accords, du type de partenaire recherché et des raisons qui poussent les entreprises à établir des accords. La seconde partie est consacrée aux objectifs prioritaires poursuivis, dans l'établissement des alliances, aux critères de succès et aux aides au succès des accords. La troisième partie traite des facteurs critiques du succès dans la gestion des alliances. 


\title{
La perception du succès des alliances stratégiques
}

\author{
Marc INGHAM* \\ Institut d'administration et de gestion \\ Université Catholique de Louvain
}

\begin{abstract}
RÉSUMÉ
Cet article présente les résultats d'une enquête-pilote sur les alliances stratégiques. II s'intéresse en particulier à la perception qu'ont les responsables d'entreprises du succès des accords. II tente de relever les différences d'opinion chez les dirigeants de PME ou de plus grandes entreprises, ou selon que les personnes interrogées appartiennent à une entreprise ayant ou non une expérience en matière d'alliances. Les propositions soumises aux répondants sont issues de la littérature récente. Dans la première partie de l'article, nous présentons la perception qu'ont les responsables d'entreprises des fonctions sur lesquelles portent en priorité les accords, du type de partenaire recherché et des raisons qui poussent les entreprises à établir des accords. La seconde partie est consacrée aux objectifs prioritaires poursuivis dans l'établissement des alliances, aux critères de succès et aux aides au succès des accords. La troisième partie traite des facteurs critiques du succès dans la gestion des alliances.
\end{abstract}

\begin{abstract}
This article presents the results of a pilot research on strategic alliances. It focuses on how managers perceive critical success factors in these agreements. It addresses questions such as - "Do they perceive alliances in a different way according to the fact that they belong to a small or medium sized company or a larger one?" - "Do they perceive such agreements differently according to the fact that they work in a business having or not a previous experience in alliances? "The variables are drawn from recent literature.
\end{abstract}

* M. Ingham est assistant à l'Institut d'administration et de gestion de l'Université Catholique de Louvain. Il enseigne la stratégie dans différents programmes universitaires et post-universitaires. Adresse : Institut d'administration et de gestion de l'Université Catholique de Louvain. 
In the first part of the paper, we address questions such as which functions are given most emphasis by companies, the type of firms with which alliances are preferred and the environmental reasons raised in support of strategic alliances. In the second part, we present the opinion managers have on the objectives they give priority to establish alliances, the success criteria and the factors facilitating successful agreements. In the third part, we develop the opinion managers have on key success factors in the management of strategic alliances.

\section{RESUMEN}

Este artículo presenta los resultados de una investigación piloto sobre las alianzas estratégicas. Particularmente, este artículo tenta sobre las percepciones que los responsables de las empresas tienen sobre el éxito de los acuerdos.

Se tenta de identificar las diferencias en las opiniones de los responsables de las PyME o de grandes empresas segun el grado en que las personas interrogadas poseen una experiencia o no en materia de alianzas. Las variables propuestas a las personas tienen como fuente diversas publicaciones recientes en el tema del management.

En la primera parte del artículo, presentamos la percepción de los responsables de las empresas de las funciones que merecen la prioritad en el caso de alianzas, tal como la investigación en conjunto, y de las razones que estimulan a las empresas a establecer estos acuerdos.

En la segunda parte, presentamos los objetivos prioritarios buscados en el establecimiento de estrategias, criterios de éxito y las ayudas para lograr dicho éxito.

La tercera y ultima parte trata sobre los factores críticos de éxito en la gestión de las alianzas.

Nous remercions messieurs Jean Mertens et Guy Van Geersdaele de la fédération d'entreprises FABRIMETAL, André d'Olne, Bruno Favaletto et Tim Willianson de l'Association Belge de Management stratégique (SCEPS) ainsi que le professeur H. Heyvaert qui nous ont facilité l'accès aux répondants. Nous remercions aussi nos collègues $Y$. De Ronge et $Y$. Delvigne. Nous remercions enfin trois lecteurs anonymes pour leurs remarques et suggestions constructives sur une première version de cet article. 


\section{Introduction}

Le thème des alliances stratégiques est largement développé et débattu dans la littérature récente. Nous présentons dans cet article l'opinion de 80 dirigeants et cadres sur l'importance des alliances, les objectifs prioritaires poursuivis par les entreprises, les motifs qui guident leurs responsables à établir de tels accords, les facteurs clés du succès de ces opérations.

Les questions à l'origine de cette enquête sont les suivantes :

- Comment les responsables d'entreprises interrogés perçoivent-ils l'importance des propositions issues de la littérature?

- Existe-t-il des différences de perception suivant qu'ils exercent leurs activités dans une grande entreprise ou une PME?

- Existe-t-il des différences de perception suivant qu'ils exercent leurs activités dans une entreprise ayant ou non une expérience en matière d'alliances?

\section{Définition des alliances}

Les alliances sont définies dans l'enquête comme étant des accords explicites, établis dans une perspective de longue durée, par lesquels des entreprises échangent, partagent ou combinent des moyens (humains, techniques, financiers) pour atteindre un ou plusieurs objectifs (assurer la rentabilité à long terme, une croissance équilibrée, réduire des risques et des délais) afin de bâtir ou maintenir des avantages et positions concurrentiels favorables.

\section{L'échantillon et la méthode}

L'échantillon compte 80 personnes dont 43 exercent leur activité dans une PME (entreprise indépendante de moins de 250 personnes) et 29 dans une grande entreprise. Huit responsables n'ont pas permis d'identifier leur entreprise, six répondants relèvent de filiales différentes d'une grande entreprise multinationale et deux grandes firmes nationales sont représentées chacune par deux répondants. Les fonctions exercées sont variées : direction générale (14 cas dont $12 \mathrm{PME})$; recherche et développement (16 cas dont 6 directeurs et $7 \mathrm{PME})$; production (19 cas dont 9 directeurs et $12 \mathrm{PME}$ ); marketing (15 cas dont 4 directeurs et $9 \mathrm{PME}$ ). La plupart des entreprises sont situées dans la partie francophone de la Belgique ou à Bruxelles et le secteur des fabrications métalliques est largement représenté. La quasi-totalité des répondants appartiennent à des entreprises industrielles, trois d'entre eux à des entreprises de service de petite dimension. 
La méthode utilisée a consisté, au départ, dans la formulation de propositions, lesquelles ont ensuite été regroupées par questions, et ce, après une analyse de la littérature ${ }^{1}$. Les réponses se situent sur une échelle à quatre positions traduisant le degré d'accord ou l'importance attribuée à la proposition. Les données ont été traitées au moyen des statistiques descriptives (moyennes, fréquences, tests sur les différences de moyennes), de l'analyse factorielle et de l'analyse discriminante en utilisant le programme Statgraphics. Les critères utilisés pour l'analyse discriminante sont le fait d'appartenir à une PME, à une grande entreprise, à une entreprise ayant ou non une expérience en matière d'alliances. L'analyse factorielle a surtout été utilisée dans une optique de confirmation, afin de vérifier les regroupements que nous avions effectués a priori entre les variables traduisant des approches différenciées des alliances. Nous retenons en général les facteurs pour lesquels les valeurs propres sont supérieures à 1 et présentons les solutions après rotation Varimax. Pour effectuer le traitement des données, nous avons formulé l'hypothèse classique voulant que les écarts existant entre les scores attribués correspondent à des distances psychologiques identiques chez les répondants. On constate que la plupart des répondants attribuent des scores élevés aux propositions formulées. C'est pourquoi nous fournissons en annexe les valeurs alpha des tests sur les différences de moyenne.

Les résultats de cette enquête ne sont pas généralisables notamment en raison du fait que les répondants ont rempli le questionnaire de façon non homogène, les uns par envoi postal, les autres en notre présence.

\section{Appréciation de l'importance du phénomène}

Bien qu'il n'existe pas de banques de données permettant de rendre compte de façon exhaustive de l'évolution du nombre d'accords de coopération, en particulier en ce qui concerne les PME, la plupart des études indiquent une augmentation du nombre d'accords depuis le début des années 1980. Différents auteurs ont relevé, pour les grandes entreprises, les secteurs et fonctions sur lesquels portent en priorité les accords ${ }^{2}$, les motifs qui poussent les entreprises à établir des alliances, le type de partenaires (taille et position concurrentielle) avec lesquels un accord est conclu de préférence, les objectifs poursuivis et le type de synergies recherchées.

1. Nous ne pouvons présenter ici l'analyse de littérature ayant servi de base aux propositions. Voir à ce sujet M. Ingham (1990).

2. Nous présentons au tableau annexe 1 une synthèse de résultats de recherches réalisée notamment à partir des travaux de Chesnais (1988), Petrella (1988), Doz dans De Woot et alii (1990), Jacquemin et Remiche (1987). 
La synthèse des résultats issus de notre échantillon est présentée au tableau 1 en annexe.

\subsection{Fonctions concernées}

Les répondants estiment que les entreprises établissent en priorité des alliances portant sur les fonctions commerciales et de recherche et développement sur les produits plutôt qu'en matière de recherche et développement sur les processus de production. La fonction approvisionnement est perçue comme étant la moins fréquemment concernée. Dans la plupart des études consultées, la fonction de recherche et développement l'emporte largement sur la production et la commercialisation.

Nous constatons que les répondants perçoivent (ce qui est assez naturel), un plus grand nombre d'accords portant sur les fonctions relevant de leur compétence au sein de l'entreprise, sauf en ce qui concerne la fonction commerciale qui est privilégiée par la plupart d'entre eux. Les dirigeants de PME ont tendance à percevoir un plus grand nombre d'alliances en production et moins d'alliances en recherche et développement sur les produits et les processus que leurs collègues de grandes entreprises, bien que les différences de moyennes soient peu significatives. Ceci pourrait être expliqué par le fait que le nombre relatif de répondants en charge de la production est plus élevé dans la catégorie PME et/ou que leur direction générale est plus fortement sensibilisée par les alliances dans cette fonction, la recherche et développement étant peut-être moins représentée en tant que fonction spécifique dans ces entreprises.

\subsection{Taille et position concurrentielle des partenaires}

L'opinion qui domine dans notre enquête est que les entreprises établissent de préférence des alliances avec des partenaires de même taille, quelle que soit la taille de leur entreprise ou la fonction sur laquelle l'accord porte en priorité. Les asymétries de taille semblent évitées, surtout pour les responsables de PME, à l'exception de la fonction de recherche et développement pour laquelle bon nombre d'entre eux estiment que les entreprises établissent des alliances avec des sociétés de plus grande taille; l'inverse étant vrai pour bon nombre de responsables de grandes entreprises. La plupart des dirigeants de PME n'estiment pas que les entreprises privilégient des relations avec de plus petits partenaires en matière commerciale. L'avis des dirigeants sur la limitation des asymétries de taille au sein des accords confirme les observations faites par différents auteurs. Nous reviendrons sur cette question dans les sections qui suivent.

En ce qui concerne la position concurrentielle des partenaires, les résultats confirment l'évidence: les relations avec les clients et les concurrents sont 
privilégiées dans la fonction commerciale, celles avec les fournisseurs dans les fonctions de production et d'approvisionnement. La recherche et développement implique de façon relativement équilibrée les trois types de partenaires.

Quoique les différences de moyenne ne soient pas très significatives, les responsables de PME pensent, plus que les répondants de plus grandes firmes, que les entreprises privilégient les relations avec leurs fournisseurs et leurs clients (accords verticaux) plutôt qu'avec leurs concurrents (accords horizontaux). Existe-t-il chez ces responsables une plus grande réticence à collaborer avec les concurrents, notamment quand il s'agit d'entreprises de plus grande taille?

\subsection{Motifs des alliances}

Les motifs qui poussent les entreprises à établir des alliances ont été mis en lumière par différents auteurs ${ }^{3}$ :

- Pour les alliances à dominante recherche et développement, les personnes interrogées estiment que l'internationalisation de la concurrence basée sur la technologie l'emporte sur la rapidité du progrès technologique et sur le montant des investissements à consentir.

- Pour les alliances à dominante production, l'utilisation optimale des capacités de production l'emporte sur la cotraitance. Notons que les responsables de PME attribuent plus d'importance à la cotraitance que leurs collègues de plus grandes entreprises; ce qui nous paraît assez naturel.

- Pour les alliances à dominante commerciale, l'internationalisation et la globalisation de la concurrence sont les principaux motifs évoqués. En général, les répondants mettent davantage l'accent sur les motifs liés au contexte de la CEE, tels que faire face à des concurrents de la CEE, renforcer les liens avec des clients établis dans la CEE, pénétrer de nouveaux marchés de la Communauté autres que ceux qui concernent les relations avec d'autres blocs européens, nord-américain ou asiatique, etc.

On peut noter toutefois l'importance accordée à la pénétration des marchés de l'Europe centrale et de l'Est. Cette orientation CEE est plus marquée chez les dirigeants de PME, en raison sans doute de la proximité de ces marchés. Il en va de même pour le développement des relations avec des clients nationaux ou régionaux, sans doute pour des raisons similaires.

3. Voir par exemple : De Woot (1988); Doz dans De Woot et alii (1990). 


\subsection{Objectifs des alliances et types de synergies recherchées}

Les répondants estiment que les objectifs de rentabilité des ventes et des investissements sont plus importants que la réduction des risques et des délais dans l'établissement d'alliances. Cette opinion est plus marquée chez les responsables de PME notamment en ce qui concerne la rentabilité d'exploitation. La réduction des risques et des délais est plus fréquemment soulignée par les responsables de la recherche et du développement que par leurs collègues d'autres fonctions.

En ce qui concerne le type de synergie recherchée en priorité par les entreprises avec les accords, l'addition des moyens techniques, humains et financiers est plus fréquemment citée que l'échange ou le partage de ces moyens notamment dans les fonctions commerciales et de production. L'échange de moyens l'emporte en recherche et développement. Il apparaît que les accords constituent un moyen d'accroître les moyens des firmes plutôt qu'à réaliser des économies. Si l'on compare ces résultats aux motifs des alliances présentés à la section précédente, il ressort que celles-ci sont perçues comme ayant un caractère « offensif » notamment en matière de production et de commercialisation. Pour les alliances RD, l'on peut suggérer que l'échange de moyens traduit la préoccupation de faire face à la complexité croissante des technologies et à la rapidité du progrès technique.

\section{Appréciation du risque et du succès des alliances}

Nous abordons dans cette section les questions relatives à la perception du risque des alliances, des critères qui permettent d'en apprécier le succès ou des variables qui peuvent constituer des aides au succès ${ }^{4}$.

\subsection{Appréciation du risque}

Différents auteurs ont mis en lumière le nombre d'échecs et les risques que comportent les alliances stratégiques à cause de la domination d'un partenaire au sein de l'accord. Les résultats de notre enquête indiquent que les responsables interrogés n'estiment pas que les alliances soient souvent un échec. Si elles apparaissent moyennement risquées, elles ne le sont pas plus que le

4. Nous nous basons dans cette section sur Buckley et Casson (1988), Kogut (1988), Schaan (1988), Harrigan (1988), Jacquemin (1986), Hamel, Doz et Prahalad (1989), Joffre et Koenig (1984), Permutter et Heenan (1988), Ohmae (1989), Lyles (1987), Verna (1988), Gomez Casseres (1987), Delvin et Blackley (1988). Pour une présentation de ces travaux ayant servi à formuler les propositions de l'enquête, voir $\mathbf{M}$. Ingham (1990). 
développement autonome. De même, si les personnes interrogées sont moyennement d'accord pour considérer que les alliances amènent un partenaire à dominer l'autre, ils ne pensent pas qu'elles soient un jeu à somme nulle. Les scores moyens enregistrés sur cette question sont significativement inférieurs à ceux qui se rapportent aux autres questions de l'enquête et il n'existe pas de différences significatives dans la perception des responsables de PME ou de grandes entreprises. Il existe cependant une différence de moyenne significative suivant l'appartenance des répondants à une entreprise ayant ou non une expérience en matière d'alliances. Les répondants qui appartiennent à une entreprise disposant d'une telle expérience perçoivent notamment un plus grand nombre de succès et de moindres risques.

\subsection{Appréciation du succès et des aides au succès}

L'appréciation de la réussite et des aides au succès des alliances stratégiques est délicate. Selon le point de vue de chaque partenaire, une alliance peut être une réussite pour l'un et s'avérer un échec pour l'autre suivant les critères retenus. De plus, la réussite d'une alliance peut être appréciée en tenant compte ou non des bénéfices indirects que peut retirer chaque partie en matière d'apprentissage et de transfert d'apprentissage à l'ensemble de l'organisation.

\subsubsection{Appréciation du succès}

Du point de vue de chaque partenaire, le succès d'une alliance peut être jugé à la contribution qu'elle apporte à l'obtention et au maintien d'avantages concurrentiels déterminants et à la mesure dans laquelle elle permet d'atteindre ses objectifs individuels.

La possibilité d'apprendre du partenaire et de transférer cet apprentissage à l'ensemble de son organisation tout en protégeant son propre savoir-faire constituent aussi des critères de succès. D'autres variables peuvent être retenues pour apprécier la réussite d'un accord. Parmi elles, citons l'atteinte des objectifs mutuels des partenaires, la durée et la stabilité de l'accord, le fait de passer plusieurs accords avec le même partenaire ou l'inexistence de conflits entre les parties.

Ces critères se rapportent à deux approches dans l'appréciation de la réussite d'une alliance. La première, que nous qualifions a priori «d'individualiste ", recouvre l'apprentissage et le transfert d'apprentissage, la protection de son savoir-faire et l'atteinte des objectifs individuels. La seconde, que nous qualifions a priori «d'harmonieuse », se réfère à l'atteinte des objectifs mutuels, aux faits qu'il n'existe pas de conflits entre les parties, que l'on passe d'autres accords avec le même partenaire ou que l'alliance dure longtemps. Les résultats de l'enquête sont fournis au tableau 2 en annexe. 
Bien que l'ensemble des critères soient jugés importants, l'atteinte des objectifs de l'alliance et en particulier des objectifs mutuels, la possibilité d'apprendre de l'autre et de transférer cet apprentissage à l'ensemble de son organisation présentent des scores moyens plus élevés. Par contre, le fait de pouvoir protéger son propre savoir-faire est le critère de réussite le moins important.

Les résultats de l'analyse discriminante ne sont pas significatifs. Il n'existe donc pas de différences de perception suivant que les répondants appartiennent ou non à une PME ou à une entreprise ayant ou non une expérience en matière d'alliances. On peut tout au plus observer une différence de moyenne significative pour les variables « durée » et " conflits " jugées plus importantes par les dirigeants de PME.

L'analyse factorielle nous amène à retenir une solution à quatre facteurs. Le premier sous-tend les variables traduisant l'approche "harmonieuse " de l'alliance : la durée de l'accord, le fait qu'il n'existe pas de conflits entre les partenaires et la multiplication des opérations avec le même partenaire. Rappelons que ces variables présentent les «meilleurs" scores dans l'analyse discriminante et sont privilégiées par les responsables de PME.

Dans l'analyse basée sur le critère d'expérience en matière d'alliance, c'est le fait qu'il n'existe pas de conflits entre les parties qui discrimine le plus et le score moyen des répondants appartenant à une entreprise non expérimentée est plus élevé.

Pour le second facteur, les critères de réussite présentant les poids les plus élevés sont l'apprentissage et son transfert à l'ensemble de l'organisation (l'atteinte des objectifs individuels chargeant plus ce facteur que les autres). Ces variables relèvent de l'approche que nous avons qualifiée d'individualiste. Le troisième facteur est relié positivement à l'atteinte des objectifs mutuels et négativement au fait de passer plusieurs accords avec le même partenaire ; le quatrième facteur étant relié essentiellement à la possibilité de protéger son savoir-faire.

Les deux facteurs principaux semblent confirmer l'existence de deux approches différenciées des alliances : la première, axée sur les relations harmonieuses entre les partenaires et qui serait privilégiée par les responsables de PME et la seconde qui traduisait des critères de réussite plus individualistes.

\subsubsection{Appréciation des aides au succès}

Les aides au succès des alliances stratégiques peuvent à nouveau être classées a priori en deux catégories. La première se réfère à la notion "d'équilibre » entre les partenaires : équilibre et complémentarité dans les contributions à l'accord et dans les compétences, équilibre dans la taille des partenaires, compatibilité culturelle entre les parties, partage du pouvoir au sein de l'alliance. La seconde 
a trait aux caractéristiques « structurelles » de l'accord : le fait qu'elle ne porte que sur une seule fonction, que son objet soit facile à réaliser, qu'il n'existe que deux parties à l'accord et que les partenaires ne soient pas concurrents.

Les résultats de l'enquête indiquent que les variables traduisant l'équilibre entre parties présentent des scores plus élevés que celles qui ont trait aux caractéristiques structurelles de l'alliance. À nouveau, les variables retenues ne permettent qu'une faible discrimination entre les PME et grandes entreprises ou selon l'expérience en matière d'alliance.

Celles qui présentent les meilleurs scores discriminants en retenant le critère de taille sont le partage du pouvoir et le fait que l'alliance ne porte que sur une seule fonction. Il semble que les dirigeants de PME accordent plus d'importance que les responsables de grandes entreprises à l'équilibre de taille, au fait que les partenaires ne soient pas concurrents et que l'accord ne porte que sur une seule fonction. Ceci confirme les résultats observés dans la première partie de l'enquête. Le partage du pouvoir est jugé plus important par les responsables de grandes entreprises.

L'analyse factorielle permet de confirmer l'existence des deux approches retenues. La solution à quatre facteurs indique que deux d'entre eux (le premier et le troisième) sous-tendent des variables liées à l'équilibre. Pour le premier, il s'agit des contributions et des tailles des partenaires; pour le second, de la complémentarité des compétences et du partage du pouvoir.

Les deux autres facteurs se rapportent aux caractéristiques structurelles de l'accord: pour le premier, il s'agit du fait qu'il ne porte que sur une seule fonction et qu'il n'implique que deux partenaires; pour le second, il s'agit du fait que l'objet de l'accord soit facile à remplir et que les partenaires ne soient pas concurrents.

\section{Facteurs clés du succès dans la gestion des alliances}

Les alliances stratégiques posent des problèmes particuliers au plan de la gestion. Nous distinguons dans l'enquête les phases de préparation, de négociation et de mise en œuvre de l'accord.

\subsection{Phase de préparation}

Les variables susceptibles de favoriser la réussite de l'alliance durant la phase de préparation peuvent être classées a priori en deux catégories. La première recouvre l'implication de la direction générale dans le processus d'élaboration 
de la stratégie, l'implication des cadres qui seront en charge de l'opération, la formation et l'information des cadres. Ces variables sont regroupées sous le vocable "ressources humaines". La seconde catégorie, qualifiée «d'analyse stratégique », recouvre la fixation d'objectifs précis, le fait de sélectionner plusieurs partenaires potentiels, l'analyse systématique des points forts et des points faibles de ces partenaires et en particulier la focalisation sur les aspects humains, techniques, financiers et sur leur position compétitive. Les résultats sont présentés au tableau 4.

Outre le fait de fixer des objectifs précis, l'implication de la direction générale est jugée plus importante que l'analyse des points forts et des points faibles des partenaires potentiels. Les différences de perception quant à l'importance des variables liées à la préparation de l'alliance au plan humain (formation et information des cadres) par rapport à la focalisation sur des aspects spécifiques sont en général peu significatives. Toutefois, les responsables de PME accordent plus d'importance que les autres répondants à l'implication de la direction générale et aux analyses stratégiques. Ceci s'explique sans doute par la taille des entreprises et la plus grande centralisation des décisions stratégiques en leur sein.

L'analyse discriminante n'offre pas de résultats significatifs.

Dans l'analyse factorielle, nous retenons trois facteurs principaux. Le premier sous-tend des variables relevant de la démarche d'analyse stratégique: analyse des points forts et des points faibles des partenaires potentiels, le fait d'en sélectionner plusieurs et, dans une moindre mesure, la fixation d'objectifs précis, ainsi que la focalisation sur les aspects financiers. Le second est relié à des variables « ressources humaines » telles que l'information, la formation et l'implication des cadres.

\subsection{Phase de négociation}

Outre le fait de prendre l'initiative de la négociation ou de détenir le pouvoir de droit au sein de l'alliance, les facteurs clés du succès retenus dans l'enquête peuvent à nouveau être regroupés à priori en deux catégories. La première traduit une approche «formalisée » de l'accord. Les variables retenues ont trait à la négociation d'un accord formel, à la fixation précise des apports de chaque partenaire, au mode de répartition des bénéfices de l'alliance et de résolution de conflits potentiels entre les parties. La seconde traduit une approche "flexible » dans la négociation : fixation des grandes lignes de l'accord, flexibilité dans la relation entre partenaires, priorité accordée au dialogue. Les résultats de l'enquête présentés au tableau 5 indiquent que les résultats moyens les plus élevés sont attribués à la fixation des apports de chaque partie, à la flexibilité de l'accord et la fixation de ses grandes lignes et des modes de résolution des 
conflits potentiels plutôt qu'à la prédiction des répartitions des bénéfices d'une manière précise ou la détention de l'initiative de la négociation, ou encore, d'un pouvoir de droit.

Les résultats de l'analyse discriminante à partir du critère d'appartenance à une PME ou à une plus grande entreprise ne sont pas significatifs. Le fait de prévoir les modes de résolution des conflits potentiels, de fixer précisément les apports de chaque partenaire, d'avoir l'initiative de la négociation et de négocier un accord formel présentent les cœfficients les plus élevés. Notons que les responsables de PME accordent plus d'importance que leurs collègues de grandes entreprises au fait de négocier les grandes lignes de l'accord et les modes de résolution des conflits potentiels. Les dirigeants de PME accordent en général moins d'importance à la «formalisation de l'accord » et à la « maîtrise du processus ". Les résultats de l'analyse discriminante selon le critère d'expérience en matière d'alliance sont plus significatifs et fournissent la prédiction de classement attendue dans $79,5 \%$ pour le groupe de répondants appartenant à une entreprise expérimentée en matière d'alliance et dans $69 \%$ des cas pour le second groupe. Les variables traduisant une approche formalisée se voient en général attribuer des scores moyens plus élevés par les répondants du second groupe. L'expérience en matière d'alliances semble atténuer la nécessité de négocier un accord formalisé. Par contre, il apparaît en général plus important aux yeux des répondants «expérimentés » d'avoir l'initiative de la négociation et de détenir un pouvoir de droit.

Dans la solution à quatre facteurs, le premier sous-tend des variables traduisant une approche basée sur la maîtrise du processus ; avoir l'initiative de la négociation et détenir un pouvoir de droit, ainsi que sur la négociation d'un accord formel. Le second se rapporte à la détermination précise du mode de répartition des bénéfices et de résolution des conflits. Les deux derniers facteurs sont reliés à des variables dont les poids les plus élevés sont de signes opposés. Dans le premier cas, il s'agit de la fixation précise des apports de chaque partie ( chargement positif) et de privilégier une approche flexible (chargement négatif). Dans le second cas, il s'agit du fait de fixer les grandes lignes de l'accord ou au contraire de négocier un accord formel. Ces résultats confirment l'évidence.

Il apparaît dans la solution à deux facteurs que la fixation des apports, la négociation d'un accord formel et le fait de prendre l'initiative de la négociation d'une part et que le fait de déterminer les grandes lignes de l'accord, d'autre part, se rapprochent des extrémités du même axe factoriel. 


\subsection{Phase de mise en cuvre de l'alliance}

Les propositions soumises aux répondants concernent le fait que ce stade est déterminant pour la réussite de l'accord et qu'il convient d'investir dans la relation avec le partenaire ou qu'au contraire à ce stade l'essentiel est joué. D'autres variables ont trait au rôle de la direction générale et des cadres, au fait de respecter l'accord formel, d'apprendre vite du partenaire et de transférer rapidement cet apprentissage à l'ensemble de l'organisation ou encore à protéger son propre savoir-faire et à contrôler l'autre partie. Les dernières propositions concernent le maintien de relations souples et d'un dialogue régulier avec le partenaire. Les résultats de l'enquête sont fournis au tableau 6.

Les responsables interrogés accordent en moyenne plus d'importance au dialogue et aux relations souples avec le partenaire. Le rôle des cadres est aussi jugé important à ce stade déterminant. Viennent ensuite le rôle de la direction générale et le fait d'investir dans la relation. Ces variables présentent des scores plus élevés que le transfert rapide des compétences, l'apprentissage, le respect de l'accord formel, le contrôle du partenaire ou la protection de son propre savoir-faire. Les résultats de l'analyse discriminante sont peu significatifs quel que soit le critère retenu. On constate toutefois que les responsables de PME ont une tendance légère à être plus sensibles que leurs collègues des grandes entreprises aux variables traduisant une relation souple avec le partenaire. Il en va de même pour les répondants appartenant à une entreprise expérimentée en matière d'alliances.

L'analyse factorielle permet de déceler cinq facteurs principaux dont certains sont difficiles à interpréter. Le premier sous-tend le fait de contrôler le partenaire, d'apprendre vite du partenaire, de transférer rapidement cet apprentissage et de respecter l'accord formel. Toutes ces variables se réfèrent à une approche individualiste dans la mise en œuvre de l'alliance. La variable qui présente le poids le plus important pour le second facteur est, qu'à ce niveau de mise en œuvre de l'accord, l'essentiel est joué. Dans la solution à deux facteurs, le fait de contrôler le partenaire, d'apprendre vite de l'autre, de transférer vite cet apprentissage et de respecter l'accord formel, tout en maintenant des relations souples et un dialogue régulier avec les partenaires, se rapprochent du même axe directionnel.

\section{Discussion des résultats}

Les résultats de notre enquête indiquent que les responsables d'entreprises interrogés confirment largement l'importance des propositions formulées au départ d'une revue de la littérature. Plusieurs résultats concernant notamment les PME nous ont cependant quelque peu surpris. 


\subsection{Importance du phénomène}

En ce qui concerne les perceptions qu'ont les personnes interrogées de l'importance du phénomène des coopérations présentées à la section 1 , nous nous attendions à voir privilégier les accords en recherche et développement et en production, conformément aux observations faites par de nombreux auteurs. Il apparaît que tant les responsables de PME que ceux de grandes entreprises attachent une plus grande importance aux accords commerciaux ainsi qu'en recherche et développement sur les produits. Au regard de ces fonctions, ce sont les motifs liés à l'internationalisation de la concurrence, et en particulier au contexte de la CEE, qui prédominent en matière commerciale. Différentes raisons peuvent être évoquées pour expliquer ce classement: la perspective de réalisation du grand marché européen de 1993 et l'ouverture des marchés de l'Europe centrale et de l'Est (lesquelles nécessitent, dans la plupart des cas, l'établissement d'alliances (entreprises conjointes) avec des partenaires locaux), la taille limitée du marché national et l'ouverture traditionnelle des entreprises belges au commerce international. Le fait que nous ayons retenu un effectif maximum de 250 personnes pour classer les entreprises dans la catégorie PME peut sans doute en partie expliquer cette sensibilité et le fait que les alliances avec des clients nationaux ou régionaux ne soient pas jugées prioritaires.

Pour les autres fonctions, nous nous attendions à ce que les responsables de PME attachent plus d'importance aux accords de production que leurs collègues de plus grandes entreprises. Ceci est peut être dû en partie aux fonctions exercées par nos répondants qui tendent à percevoir un plus grand nombre d'alliances dans leur domaine, mais aussi au fait que l'orientation prioritaire des responsables de PME industrielles vers les problèmes de production demeure importante et que la fonction spécifique de recherche et développement est encore souvent embryonnaire dans les PME et fortement liée à la production. Ceci peut expliquer le fait que les responsables de PME ont tendance à accorder plus d'importance que les autres répondants aux alliances en matière de recherche et développement sur les processus.

Le fait que les répondants privilégient les relations avec des entreprises de tailles comparables ou évitent les fortes asymétries de taille ne nous a pas surpris. Nous retrouvons cette sensibilité dans l'analyse des aides au succès des alliances traduisant l'équilibre entre les partenaires. Cet équilibre de taille peut favoriser la compatibilité « culturelle » entre parties et faciliter le dialogue entre elles. Elle permettrait aussi de limiter les risques de domination d'un partenaire au sein de l'accord. Ce risque est toutefois jugé comme étant relativement faible par les répondants.

Le fait que les responsables de PME soient quelque peu plus sensibles que leurs homologues de grandes entreprises aux alliances établies avec des fournisseurs ou des clients et fassent preuve de plus de réticence à coopérer 
avec des concurrents directs ou potentiels tient peut-être aux risques de domination, notamment quand il s'agit d'accords avec des partenaires de grande taille. Notons aussi que ces dirigeants de PME ont tendance à être plus sensibles aux variables traduisant une approche harmonieuse des alliances quand il s'agit d'apprécier les critères de succès des accords et les facteurs de succès dans la phase de mise en œuvre de l'accord (voir section 3). Ces relations sont sans doute plus faciles à établir et à maintenir dans le cas d'alliances verticales.

\subsection{Appréciation du risque}

Les résultats concernant l'appréciation des risques des alliances stratégiques nous ont particulièrement surpris. La plupart des études consacrées aux alliances insistent sur le nombre d'échecs des opérations et sur le fait que les alliances constituent, dans un grand nombre de cas, un second choix par rapport au développement autonome. De même, les ris̀'ques de voir les accords ne bénéficier qu'à un seul partenaire (jeu à somme nulle) ou à tout le moins de voir l'un d'entre eux dominer l'autre sont développés dans la littérature. En poussant le raisonnement à l'extrême, l'alliance entre concurrents directs ou potentiels pourrait être un « cheval de Troie » permettant à un partenaire de dominer l'autre plus rapidement que par le jeu normal de la concurrence.

Or, comme nous l'avons vu à la section 2 , nos répondants attribuent aux propositions sur ce sujet des scores moyens inférieurs à la plupart des autres variables. Ils ne croient pas que les alliances soient souvent un échec et, si ils sont moyennement d'accord pour les considérer comme étant risquées, ils sont encore moins d'accord sur le fait qu'elles soient plus risquées que le développement autonome. De même, ils ne sont que moyennement d'accord pour considérer que les alliances amènent un partenaire à dominer l'autre et encore moins qu'elles représentent des jeux dans lesquels ce que l'un gagne l'autre le perd.

Nous constatons aussi que sur 46 répondants déclarant appartenir à une entreprise ayant conclu dans les cinq années une ou plusieurs alliances, 25 affirment que leur entreprise à l'intention d'en établir de nouvelles d'ici cinq ans, 12 envisagent cette possibilité et aucun ne la rejette. Ces résultats sont sans doute le signe d'une satisfaction quant aux résultats obtenus. Parmi les 21 autres personnes ayant répondu à cette question, 6 déclarent que leur entreprise n'a pas l'intention d'établir d'alliances dans ces délais, 7 autres envisagent la possibilité d'en établir et 6 personnes déclarent que leur entreprise passera un ou plusieurs accords d'ici cinq ans, 2 cas n'ont pu être traités (répondants d'une même entreprise ayant des avis divergents). Ces résultats tendent à indiquer une satisfaction de la part des responsables d'entreprises expérimentés. On remarque que leur nombre est relativement élevé dans l'échantillon entrainant peutêtre un biais dans l'enquête. Les résultats indiquent aussi que rares sont les répondants responsables de PME qui excluent la possibilité de passer des accords. 


\subsection{Critères de succès et aides au succès}

Les résultats concernant les critères de succès nous ont particulièrement intéressé. Nous avions relevé deux courants de pensée concernant les alliances qui, sans être contradictoires, présentent des vues contrastées. Dans le premier courant, l'accent est mis sur le point de vue de l'une des parties à l'accord qui adopterait des critères essentiellement basés sur les avantages individuels retirés de l'alliance à l'égard de l'atteinte des objectifs individuels, de l'apprentissage et de la protection du savoir-faire : approche que nous avions qualifiée d'individualiste. L'autre approche, qualifiée d'harmonieuse, met l'accent sur l'atteinte des objectifs mutuels, la durée de l'accord, la non-existence de conflits entre partenaires et le fait de passer des accords avec d'autres partenaires.

Tout en confirmant l'existence de ces approches, il est frappant de constater que les répondants ne privilégient pas systématiquement l'une d'entre elles dans leur appréciation des critères de succès ; les responsables de PME étant plus sensibles que les autres aux critères traduisant l'harmonie entre les partenaires : durée de l'accord et non-existence de conflits. Cette sensibilité particulière aux conflits est confirmée quand ils évaluent les facteurs clés du succès durant la phase de négociation de l'accord.

La possibilité d'apprendre de l'autre et de transférer cet apprentissage à l'ensemble de l'organisation constituent des critères jugés importants, alors que la protection du propre savoir-faire ne l'est pas. Ces variables ont été utilisées à nouveau pour apprécier les facteurs clés du succès durant la phase de mise en œuvre de l'alliance. Il est intéressant de constater que dans cette mise en œuvre, l'apprentissage et son transfert revêtent alors une importance moindre aux yeux des répondants (tant de façon absolue que relativement aux autres facteurs de succès). On peut aussi être surpris du peu d'importance accordée à la protection du savoir-faire par nos répondants en tant que critère de réussite ou de facteur clé du succès durant la phase de mise en œuvre. Ceci pourrait indiquer une attitude confiante de ces responsables tant en ce qui concerne le fait de disposer de compétences distinctives qui peuvent être protégées « naturellement » que vis à vis du partenaire. Il se peut aussi qu'ils estiment que leurs compétences distinctives, notamment en matière technique, soient faibles et de ce fait ne doivent pas être protégées. La première explication serait renforcée par l'importance accordée à la fixation précise des apports des parties durant la phase de négociation de l'accord, par l'importance accordée à l'équilibre dans les contributions et à la complémentarité dans les compétences des parties à l'accord (aides au succès), ces variables présentant des scores moyens très élevés.

Pour ce qui est des aides au succès, le résultat qui nous semble le plus intéressant concerne l'accent mis par les répondants sur l'équilibre entre les partenaires plutôt que sur les caractéristiques structurelles de l'accord. Les 
responsables de PME sont cependant plus sensibles que les autres à ces caractéristiques structurelles, en raison sans doute de la taille de leur entreprise et de leur plus grande réticence à établir des accords avec des concurrents.

\section{Gestion de l'alliance}

Durant la phase de préparation, les répondants combinent des facteurs clés de succès liés aux ressources humaines et à l'analyse stratégique, ces derniers aspects étant plus soulignés par les responsables de PME. Ceci peut paraître surprenant étant donné les moyens sans doute plus limités dont ils disposent pour réaliser ces analyses.

Des pistes d'explication peuvent être recherchées dans la taille de ces entreprises qui ne disposent que d'un nombre limité de cadres et où les décisions stratégiques sont nécessairement plus centralisées. Les répondants de plus grandes entreprises étant pour la plupart des cadres, il peut paraître normal qu'ils mettent davantage l'accent sur la formation, l'information et l'implication de ceux qui seront en charge de l'opération dès l'élaboration de la stratégie d'alliance.

Durant la phase de négociation, on constate que ce sont les variables traduisant une approche flexible de l'accord qui sont privilégiées par les répondants et en particulier par les responsables de PME, les responsables de grandes entreprises étant plus sensibles à la maîtrise du processus. Cette légère différence dans l'importance accordée à l'harmonie se retrouve aussi durant la phase de mise en œuvre de l'accord.

Au cours de cette phase, les répondants et en particulier les responsables de PME mettent clairement l'accent sur le maintien de relations souples et d'un dialogue régulier entre les partenaires.

D'une façon générale, les résultats de notre enquête suggèrent l'existence, chez les personnes interrogées, d'une confiance eu égard aux risques des alliances. Leurs perceptions paraissent influencées par des approches traduisant un comportement cooperatif entre les parties plutôt qu'un comportement individualiste, un comportement flexible plutôt que rigide.

Ces résultats nous paraissent intéressant dans la mesure où un courant dominant - axé sur la maîtrise du processus, l'obtention du pouvoir, l'apprentissage rapide, la prudence, voire la méfiance vis à vis du partenaire, le contrôle du partenaire, le respect des accords formels -, nous était apparu dans notre revue de littérature, notamment nord-américaine.

Le lecteur peut être surpris par le caractère peu significatif des résultats des analyses discriminantes. Ceci peut être dû au choix des variables que nous avons effectué et à une "neutralisation " dans les fonctions discriminantes ou 
encore, au fait que les répondants confirment l'importance des propositions en leur attribuant des scores élevés. La taille de l'entreprise à laquelle appartient le répondant n'influence pas de façon systématique son jugement. Tout au plus, existe-t-il des différences de perception sur des variables prises isolément ; ces différences étant confirmées par le recours à d'autres variables isolées. Les raisons tiennent peut-être au critère d'emploi que nous avons retenu pour définir la taille des entreprises ou encore à la fonction exercée par les responsables interrogés au sein des grandes entreprises.

\section{Conclusion}

En général, les responsables d'entreprises interrogés dans notre enquête confirment l'importance des propositions formulées au départ dans la littérature. Ils mettent en lumière l'importance des alliances portant sur les fonctions commerciales et de recherche et développement sur les produits ainsi que des motifs liés à l'internationalisation de la concurrence, en particulier dans le contexte de la CEE. Ces alliances revêtent un caractère " offensif » et permettent aux entreprises d'additionner des moyens humains techniques et financiers notamment pour prendre position sur de nouveaux marchés. Les entreprises semblent, à leurs yeux, privilégier les alliances avec des partenaires de même taille. Les dirigeants de PME insistent plus sur l'importance des alliances avec des fournisseurs et des clients qu'avec des concurrents. Bien que les différences de perception entre responsables de PME et de plus grandes entreprises soient peu significatives dans l'ensemble de l'enquête et que la taille de l'entreprise ne semblent pas (ou peu) influencer les perceptions des répondants, les résultats suggèrent que les dirigeants de PME mettent d'avantage l'accent sur les objectifs de rentabilité poursuivis au moyen de l'alliance. Ces dirigeants ont aussi tendance à mettre l'accent sur les variables traduisant des relations harmonieuses avec les partenaires pour apprécier la réussite d'une alliance ou les facteurs clés du succès dans la gestion de l'alliance. Ils mettent aussi davantage l'accent sur les variables liées à l'équilibre et aux relations souples avec le partenaire quand ils apprécient les aides au succès des alliances ou les facteurs clés du succès dans leur gestion. 


\section{Bibliographie}

BResser, R. (1988), "Matching collective and competitive strategies », Strategic Management Journal, vol. 9, août, p. 375-385.

BuCKLEY, P. et M. CASSON (1988), «A theory of cooperation in international business », MIR special issue, p. 19-37.

DATTA, D. (1988), «International joint ventures : a framework for analysis », Journal of General Management, vol. 14, hiver, p. 78-91.

DE Woot, P. (1988), Les entreprises de haute technologie et l'Europe, Paris, Economica.

DE Woot, P., Y. Doz, P. Haspeslagh et G. Lorenzoni (1990), « Les enjeux de la croissance externe ", Projet Penelope II, CEE Fast .

Delvin, G. et M. Blackley (1988), « Strategic alliances : guidelines for success », Long Range Planning, vol. 21, octobre, p. 18-23.

Doz, Y. «Technology partnerships between larger and smaller firms: some critical issues », International Studies of Management and Organization, vol. XVIII, $n^{\circ} 4$, p. 31-57.

Dussauge, P., B. Garette et B. Ramanantsoa (1988), «Stratégies relationnelles et stratégies d'alliances technologiques », Revue Française de Gestion, mars-avrilmai, p. 7-19.

GERINGER, J.H. (1988), « Selection of partners for international joint ventures », Business Quarterly, automne, p. 31-36.

Gomes-CAsseres, B. (1987), «Joint venture instability : is it a problem ? », Columbia Journal of World Business, été, p. 97-101.

GoRBIs, M. et K. YoRK (1988), « Strategic partnership : a new corporate response », SRI International Report , $\mathrm{n}^{\circ} 730$.

Hamel, G., Y. Doz et C.K. Prahalad (1989), « Collaborate with your competitors and win », Harvard Business Review, janvier-février, p. 113-139.

HARRIGAN, K.R. (1987), "Strategic alliances : their new role in global competition », Columbia Journal of World Business, été, p. 67-69.

HARRIGAN, K.R. (1988), « Strategic alliances and partner asymetries », MIR special issue, p. 53-72.

INGHAM, M. (1990), « Approche stratégique des alliances internationales », Gestion 2000, $n^{\circ} 5$, octobre, p. 97-129.

INGHAM, M. (1991b.), « Aspects stratégiques de la gestion des filiales communes » à paraître dans A. QUINTART et Y. DE RONGE. La filialisation; stratégie et gestion, Bruxelles, De Bœck.

InghaM, M. et L. TEMPELs (1991a), «Strategic alliances involving small and medium sized firms in the European context », Workshop Paper. IAG - UCL Forthcoming.

JofFre, P. et G. KENIG (1984), «Stratégies de coopération et d'alliances inter-entreprises », Enseignement et Gestion nouvelle, série automne.

Johnston, R. et P. LAWRence (1988), « Beyond vertical integration : the rise of value added partnership », Harvard Business Review, juillet-août, p. 94-101.

KILLING (1982), «How to make a global joint venture work », Harvard Business Review, réimpression 82310 , mai-juin, p. 120-127. 
Kogut, B. (1988a), «The study of the life cycle of joint ventures », MIR special issue, p. 39-49.

Kogut, B. (1988b), «Joint ventures : theoretical and empirical perspective », Strategic Management Journal, vol. 9, juillet-août, p. 319-322.

Lorange, P. et Probst (1987), «Joint ventures as self organizing systems : a key to successful joint venture design and implementation », Columbia Journal of World Business, été, p. 71-77.

LyLes, M. (1987), « Common mistakes of joint venture experienced firms », Columbia Journal of World Business, été, p. 19-37.

Ohmae, K. (1989), « The global logic of strategic alliances », Harvard Business Review, mars-avril, p. 136-152.

SCHAAN, J.L. (1988), « How to control a joint venture even as a minority partner », Journal of General Management, vol. 14, p. 4-16.

VERNA, J. (1988), «Comment assurer le succès des filiales communes », Revue Française de Gestion, novembre-décembre. 
Annexes 
TABleau 1

\begin{tabular}{|c|c|c|c|c|}
\hline Fonctions concernées & Taille des partenaires & $\begin{array}{l}\text { Types de synergies } \\
\text { (recherchées) }\end{array}$ & $\begin{array}{l}\text { Position } \\
\text { concurrentielle } \\
\text { des partenaires }\end{array}$ & Motifs des alliances \\
\hline $\begin{array}{l}\text { RDPROD } \\
n=80 \text { Moy. }=3,06 \\
\text { Dév. stand. }=0,86 \\
\text { Fréq. relat. score 2: } 0,15 \\
\text { score 3: } 0,45 \\
\text { score 4: } 0,34\end{array}$ & $\begin{array}{l}n=\text { nombre de citations de } \\
\quad \text { scores } 3 \text { ou } 4 \\
\text { RDPROD } \\
\text { bcp + grand: } 8,+ \text { grand: } 15 \\
\text { Identique: } 23 \\
\text { + petite: } 16 \text { bcp + petite: } 2 \\
\text { Indiffér.: } 9 \\
\qquad S=73\end{array}$ & $\begin{array}{l}n=\begin{array}{c}\text { nombre de } \\
\text { citations }\end{array} \\
\text { RDPROD } \\
\text { Partage } n=21 \\
\text { Echange } n=31 \\
\text { Addition } n=28\end{array}$ & $\begin{array}{l}n=\text { nombre de } \\
\quad \text { citations } \\
\text { RDPROD } \\
\text { Concurr.: } n=28 \\
\text { Score Moy.: } 2,3 \\
\text { Clients: } n=26 \\
\text { Score Moy.: } 3,2 \\
\text { Fourniss.: } n=29 \\
\text { Score Moy.: } 2,9\end{array}$ & $\begin{array}{l}\text { RDPROD et RDPROC } \\
\text { INTERTECH: Moy.: } 3,42-\text { Dév. } \\
\text { stand.: } 0,12 \\
\text { Fréq. relat. } \\
\text { score 3: } 0,30 \\
\text { score 4: } 0,62 \\
\text { RAPTECH: Moy.: } 3,26 \text { - Dév. } \\
\text { stand.: } 0,66\end{array}$ \\
\hline $\begin{array}{l}\text { RDPROC } \\
n=80 \text { Moy. }=2,71 \\
\text { DS =0,94 } \\
\text { Fréq. relat. } \\
\\
\text { score 2: } 0,29 \\
\text { score 3: } 0,375 \\
\text { score 4: } 0,225\end{array}$ & $\begin{array}{l}\text { RDPROC } \\
\text { bcp + grand: } 8,+ \text { grand: } 16 \\
\text { Identique: } 27 \text {, petite } 11 \\
\text { bcp + petite: } 3 \\
\text { Indiffér.: } 6 \\
\qquad S=72\end{array}$ & $\begin{array}{l}\text { RDPROC } \\
\text { Partage } n=14 \\
\text { Echange } n=36 \\
\text { Addition } n=23\end{array}$ & $\begin{array}{l}\text { RDPROC } \\
\text { Concurr.: } n=25 \\
\text { Score Moy:: } 2,1 \\
\text { clients: } n=21 \\
\text { Score Moy.: 2,4 } \\
\text { Fourniss.: } n=24 \\
\text { Score Moy.: 2,7 }\end{array}$ & 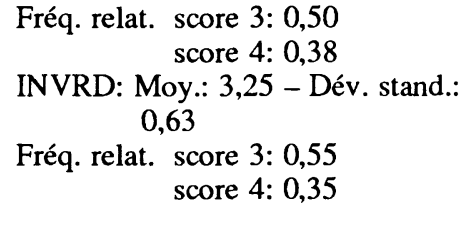 \\
\hline $\begin{array}{l}\text { PROD } \\
n=78 \text { Moy. }=2,58 \\
\text { DS =0,87 } \\
\text { Fréq. relat. } \\
\\
\quad \text { score 2: } 0,33 \\
\text { score 3: } 0,41 \\
\text { score 4: } 0,14\end{array}$ & $\begin{array}{l}\text { PROD } \\
\text { bcp + grand: } 2,+ \text { grand: } 11 \\
\text { Identique: } 36,+ \text { petite: } 14 \\
\text { bcp + petite: } 4 \\
\text { Indiffér.: } 4 \quad S=71\end{array}$ & $\begin{array}{l}\text { PROD } \\
\text { Partage } n=21 \\
\text { Echange } n=7 \\
\text { Addition } n=41\end{array}$ & $\begin{array}{l}\text { PROD } \\
\text { Concurr.: } n=23 \\
\text { Score Moy.: } 2,1 \\
\text { Clients: } n=22 \\
\text { Score Moy.: } 2,1 \\
\text { Fourniss.: } n=32 \\
\text { Score Moy.: } 3,1\end{array}$ & $\begin{array}{ll}\text { CAPACITE: Moy.: } 3,16-\text { Dév. } \\
& \text { stand.: } 0,86 \\
\text { Fréq. relat. } & \text { score 3: } 0 \\
& \text { score 4: } \\
\text { COTRAIT: } & \text { Moy.: 2,95 - Dév. } \\
& \text { stand.: 0,74 } \\
\text { Fréq. relat. } & \text { score 3: } 0,33 \\
& \text { score 4: } 0,43\end{array}$ \\
\hline
\end{tabular}


TABLEAU 1 (suite)

\begin{tabular}{|c|c|c|c|c|}
\hline Fonctions concernées & Taille des partenaires & $\begin{array}{l}\text { Types de synergies } \\
\text { (recherchées) }\end{array}$ & $\begin{array}{l}\text { Position } \\
\text { concurrentielle } \\
\text { des partenaires }\end{array}$ & Motifs des alliances \\
\hline \multirow[t]{2}{*}{$\begin{array}{l}\text { COMM } \\
n=79 \text { Moy. }=3,09 \\
\text { DS: } 0,94 \\
\text { Fréq. relat. } \\
\\
\text { score 2: } 0,14 \\
\text { score 3: } 0,37 \\
\text { score 4: } 0,40\end{array}$} & \multirow[t]{2}{*}{$\begin{array}{l}\text { COMM } \\
\text { bcp + grand: } 4,+ \text { grand: } 19 \\
\text { Identique: } 29,+ \text { petite: } 11 \\
\text { bcp + petite: } 1 \\
\text { Indiffér.: } 9 \\
\qquad S=73\end{array}$} & \multirow[t]{2}{*}{$\begin{array}{l}\text { COMM } \\
\text { Partage } n=20 \\
\text { Echange } n=20 \\
\text { Addition } n=40\end{array}$} & \multirow[t]{2}{*}{$\begin{array}{l}\text { COMM } \\
\text { Concurr.: } n=31 \\
\text { Score Moy.: } 2,6 \\
\text { Clients: } n=30 \\
\text { Score Moy.: } 2,8 \\
\text { Fourniss.: } n=22 \\
\text { Score Moy.: } 2,45\end{array}$} & $\begin{array}{l}\text { COMM } \\
\text { INTERCONC: Moy.: 3,82 DS }=0,7, n=77 \\
\text { CONCEURO: Moy.: 3,28 DS }=0,75, n=72 \\
\text { CONCUS: Moy.: } 2,77 \text { DS }=0,82, n=65 \\
\text { CONCJAP: Moy.: } 2,91 \text { DS }=0,82, n=65 \\
\text { MARCEE: Moy.: 3,45 DS }=0,68, n=76 \\
\text { MAREST: Moy.; 3,08, DS }=0,86, n=70 \\
\text { MARNORD: Moy.: } 2,92 \text { DS }=0,79, n=69 \\
\text { MARUS: Moy.: } 2,90 \text { DS }=0,84, n=66 \\
\text { MARAS: Moy.: 2,67, DS: } 0,92, n=68\end{array}$ \\
\hline & & & & $\begin{array}{l}\text { CLIETR: Moy.: 2,76 DS 0,79, } n=65 \\
\text { CLICEE: Moy.: 2,89 DS 0,78, } n=62 \\
\text { CLINAT: Moy.: } 2,83 \text { DS } 0,96, n=62 \\
\text { CLIREG: Moy. : 2,66, DS } 1,06, n=63\end{array}$ \\
\hline $\begin{array}{l}\text { APPROV } \\
n=78 \\
\text { Moy. }=2,51 \\
\text { DS }=0,95 \\
\text { Fréq. relat. } \\
\qquad \begin{array}{l}\text { score } 2=0,30 \\
\text { score } 3=0,37 \\
\text { score } 4=0,15\end{array}\end{array}$ & $\begin{array}{l}\text { bcp + grand: } 3+\text { grand: } 15 \\
\text { Identique: } 22,+ \text { petite: } 10 \\
\text { bcp + petite: } 5 \\
\text { Indiffér.: } 15\end{array}$ & $\begin{array}{l}\text { Partage } n=22 \\
\text { Échange } n=10 \\
\text { Addition } n=35\end{array}$ & $\begin{array}{l}\text { Concurr.: } n=13 \\
\text { Score: } 2,5 \\
\text { Clients: } n=12 \\
\text { Score: } 2,3 \\
\text { Fourniss.: } n=23 \\
\text { Score: } 2,1\end{array}$ & $\begin{array}{l}\text { REDUDEL: Moy.: } 3,01 \text { DS }=0,94, n=67 \\
\text { REDOSTO: Moy.: 2,89 DS }=0,88, n=66 \\
\text { AUGFREQ: Moy.: } 2,70 \text { DS }=0,80, n=66\end{array}$ \\
\hline
\end{tabular}




\section{LISTE DES VARIABLES}

TABLEAU 1

Fonctions concernées et motifs des alliances

\begin{tabular}{ll}
\hline RDPROD & Recherche et développement sur les produits \\
RDPROC & Recherche et développement sur les processus \\
PROD & Production \\
COMM & Marketing \\
APPROV & Approvisionnement \\
INTERTECH & Internationalisation de la concurrence \\
RAPTECH & Rapidité du progrès technique \\
INVRD & Montant des investissements en RD \\
CAPACITE & Utilisation optimale des capacités de production \\
COTRAIT & Cotraitance \\
INTERCONC & Internationalisation et globalisation de la concurrence \\
CONCEURO & Faire face à des concurrents européens \\
CONCUS & Faire face à des concurrents nord-américains \\
CONCJAP & Faire face à des concurrents japonais \\
MARCEE & Prendre position sur des marchés de la CEE \\
MARES & Prendre position sur des marchés de l'Europe de l'Est \\
MARNORD & Prendre position sur des marchés de l'Europe du Nord \\
MARUS & Prendre position sur des marchés nord-américains \\
MARAS & Prendre position sur des marchés asiatiques \\
CLIETR & Développer des relations avec des clients étrangers \\
CLICEE & Développer des relations avec des clients de la CEE \\
CLINAT & Développer des relations avec des clients nationaux \\
CLIREG & Développer des relations avec des clients régionaux \\
REDUDEL & Réduire les délais d'approvisionnement \\
REDOSTO & Réduire les stocks \\
AUGFREQ & Augmenter la fréquence des approvisionnements \\
\hline
\end{tabular}


TABLEAU 2

Critères de réussite des alliances

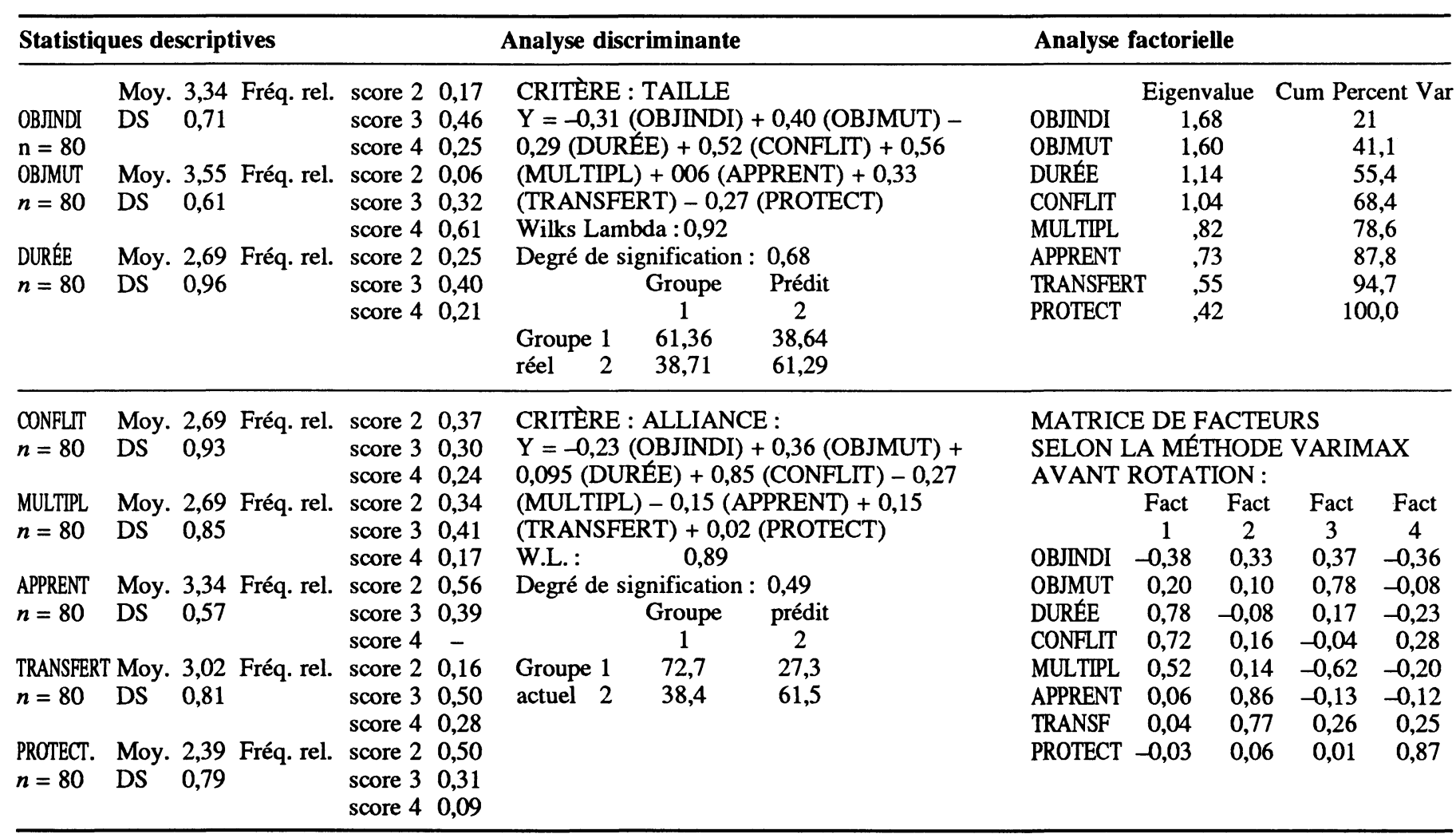


GRAPHIQUE DU TABLEAU 2

Diagramme des variables dans l'espace des facteurs 1 et 2

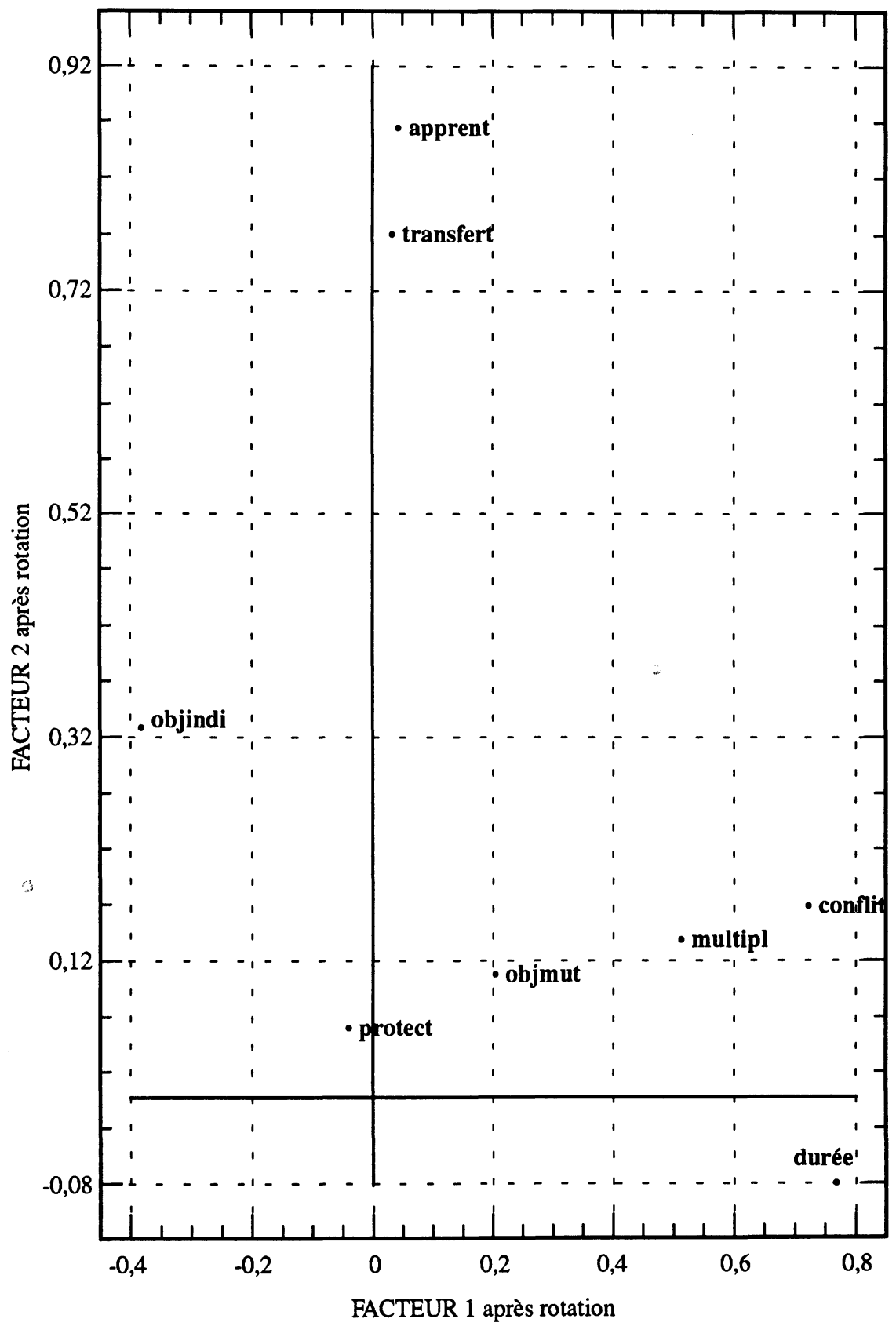

Revue internationale P.M.E., vol. 4, ${ }^{\circ}$ 2, 1991 


\section{LISTE DES VARIABLES}

TABLEAU 2

\section{Appréciation du succès des alliances}

\begin{tabular}{ll}
\hline OBJINDI & Mon entreprise atteint ses objectifs \\
OBJMUT & Les partenaires atteignent leurs objectifs \\
DUREE & L'alliance dure longtemps \\
CONFLIT & Il n'y a pas de conflit entre les parties \\
MULTIPL & D'autres accords sont passés avec le même partenaire \\
APPRENT & On apprend de l'autre \\
TRANSFERT & $\begin{array}{l}\text { On peut transférer les compétences du partenaire à l'ensemble } \\
\text { de l'organisation }\end{array}$ \\
PROTECT & On peut protéger son savoir-faire
\end{tabular}


TABLEAU 3

Aides au succès des alliances

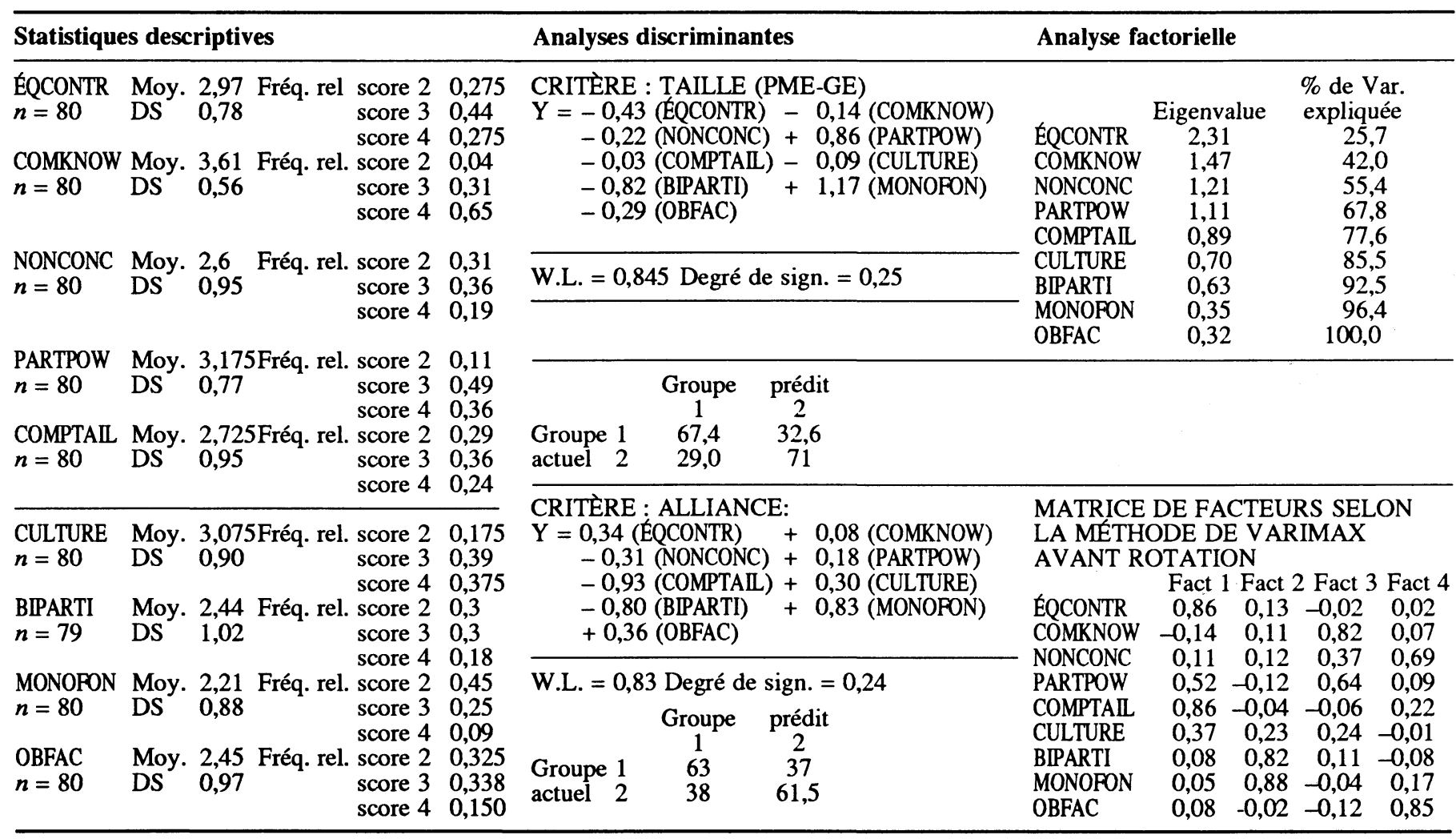


GRaPHQUE DU TABleAU 3

Diagramme des variables dans l'espace des facteurs 1 et 2

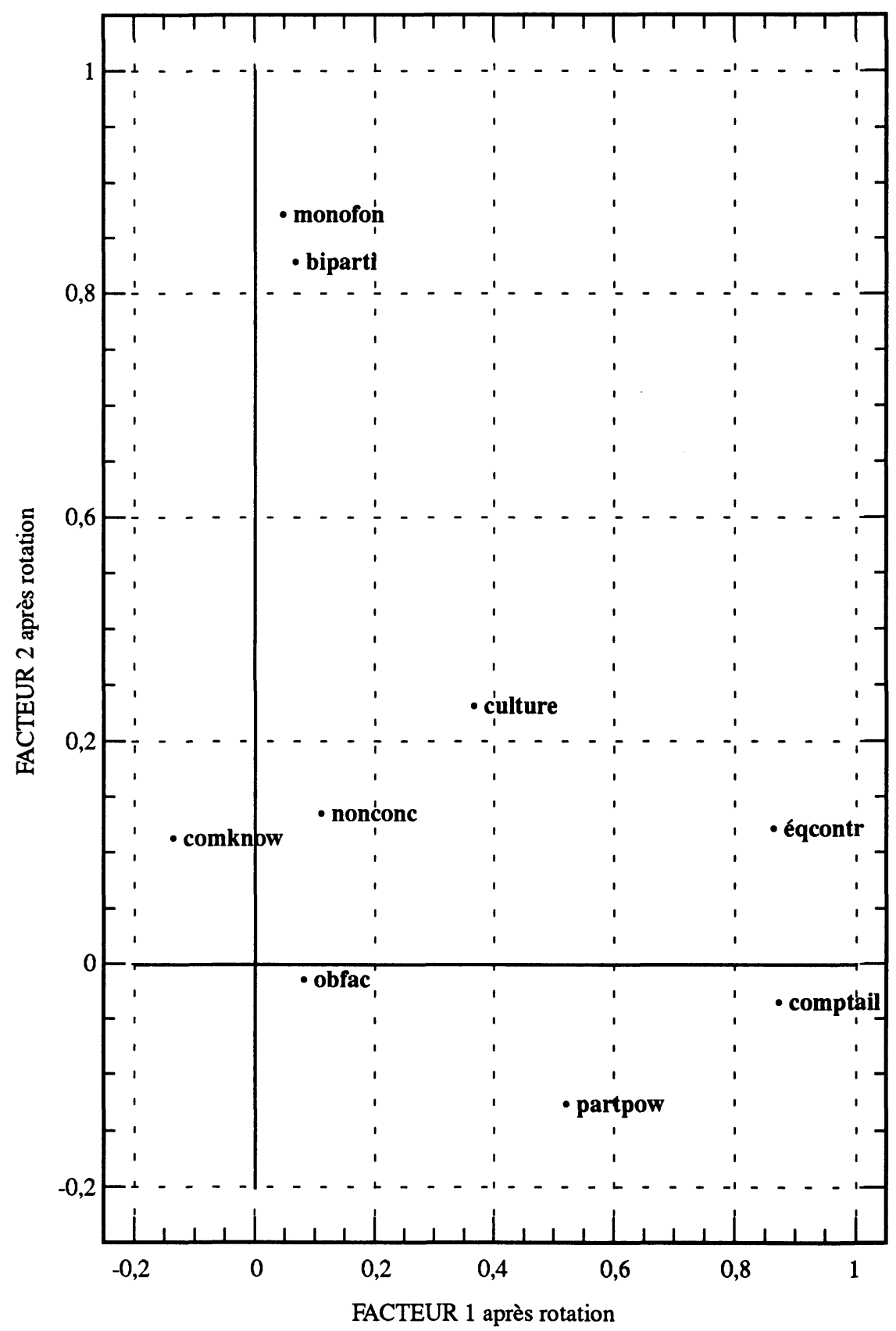

Revue internationale P.M.E., vol. 4, $\mathrm{n}^{\circ}$ 2, 1991 


\section{LISTE DES VARIABLES}

\section{TABLEAU 3}

\section{Aides au succès des alliances}

EQCONTR Les contributions des partenaires sont équilibrées

COMKNOW Les compétences des partenaires sont complémentaires

NONCONC Les partenaires ne sont pas concurrents

PARTPOW Le pouvoir est partagé au sein de l'accord

COMPTAIL Les tailles des partenaires sont comparables

CULTURE La compatibilité culturelle entre les partenaires est forte

BIPARTI Il n'y a que deux parties à l'accord

MONOFON L'accord ne porte que sur une fonction

OBJFAC L'objet de l'accord est facile à remplir 
TABLEAU 4

Gestion de l'alliance

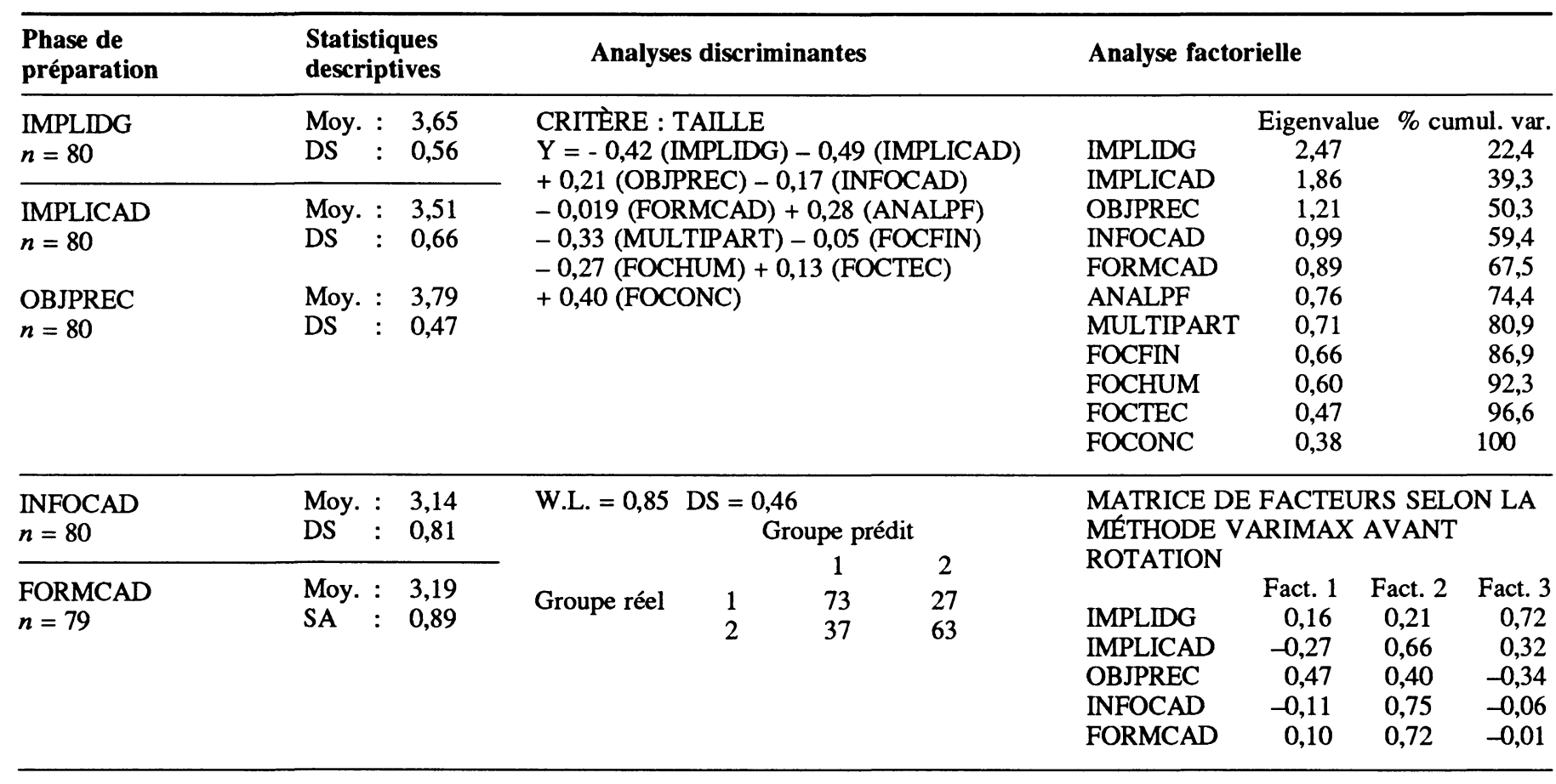


TABLEAU 4 (suite)

Gestion de l'alliance

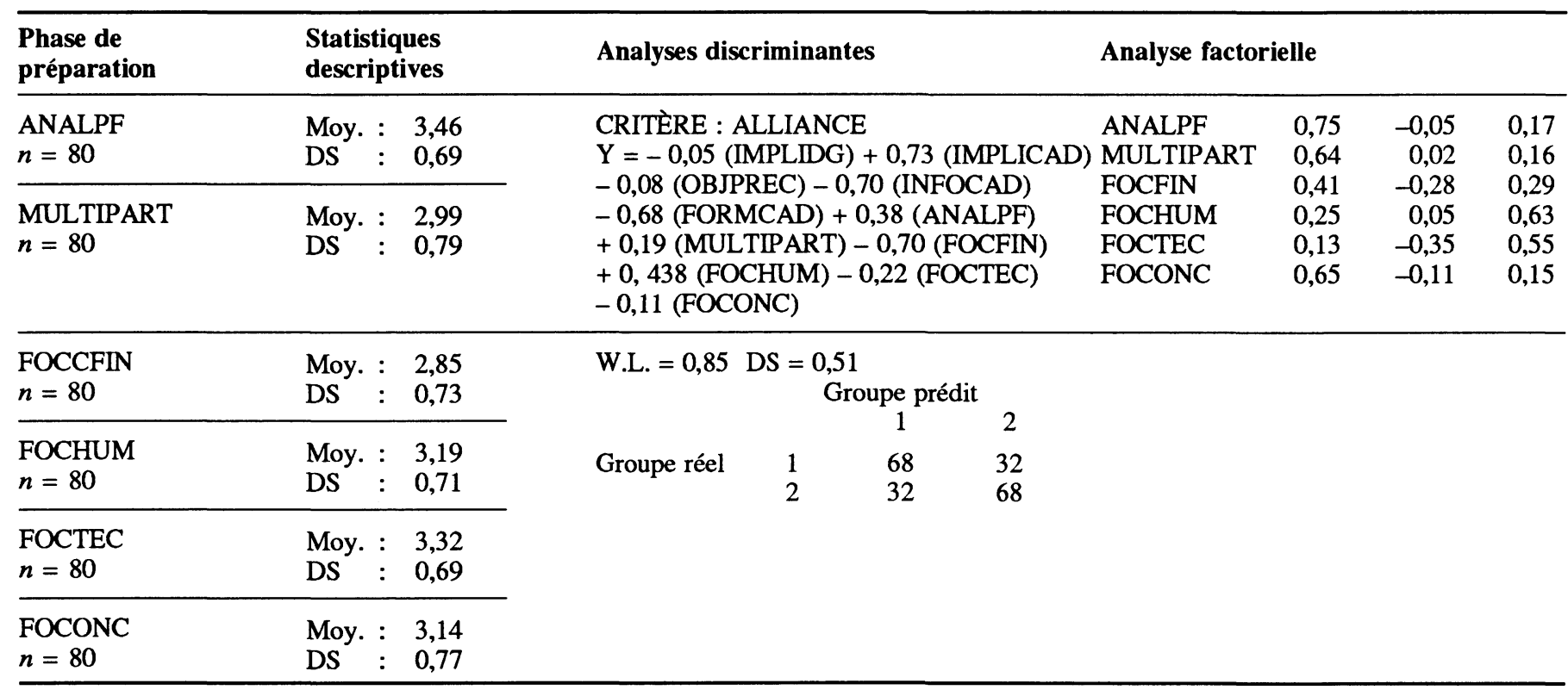


GRAPHIQUE DU TABLEAU 4

Diagramme des variables dans l'espace des facteurs 1 et 2

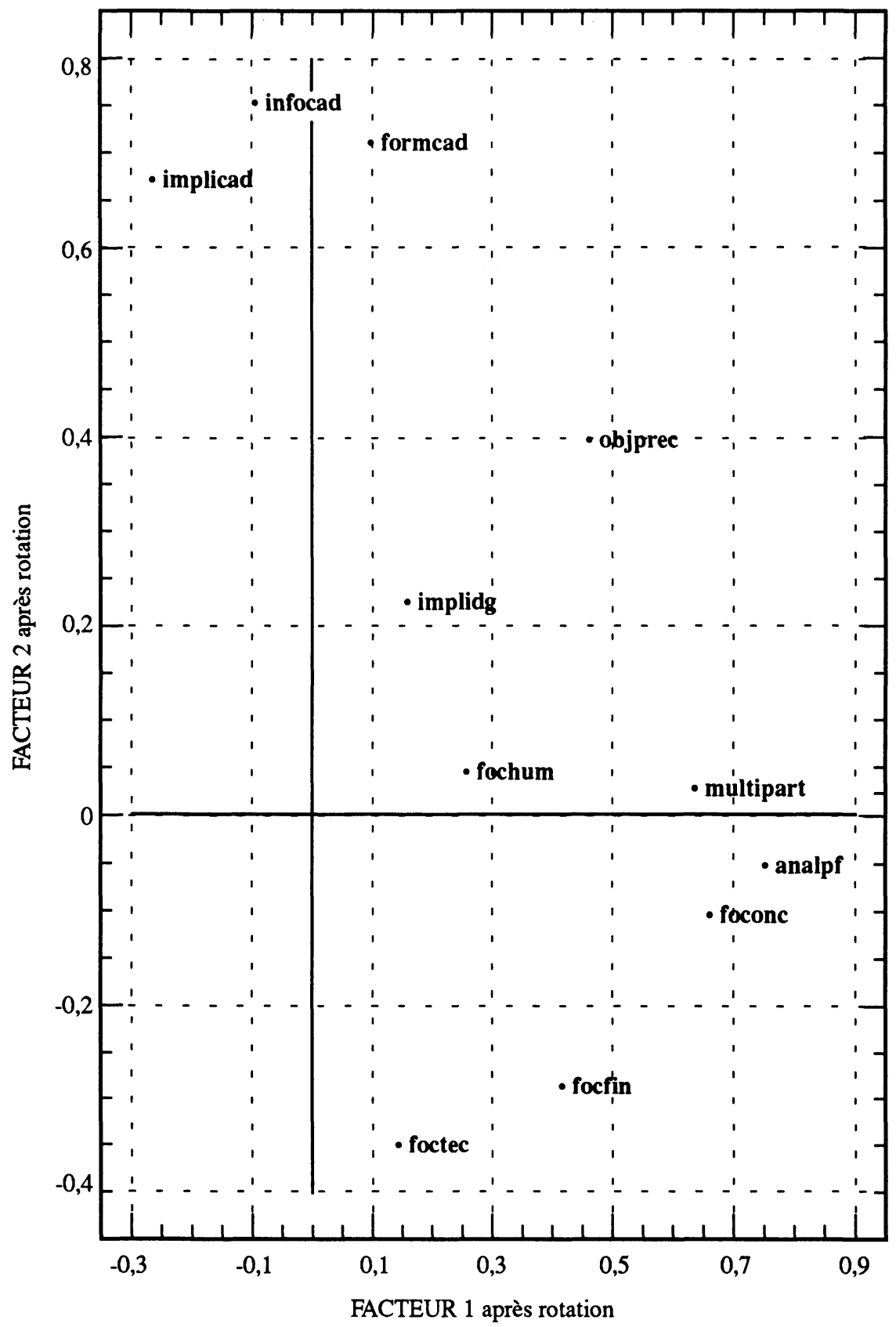

Revue internationale P.M.E., vol. 4, $\mathrm{n}^{\circ}$ 2, 1991 


\section{LISTE DES VARIABLES}

\section{TABLEAU 24}

\section{Gestion de l'alliance - phase de préparation}

IMPLIDG Implication de la direction générale dans le processus

IMPLICAD Implication des cadres qui seront en charge de l'opération

OBJPREC Les objectifs de l'accord sont fixés avec précision

INFOCAD Information des cadres qui seront en charge de l'opération

FORMCAD Formation des cadres

ANALPF Analyse des points forts et des points faibles des partenaires potentiels

MULTIPART Sélection de plusieurs partenaires potentiels

FOCFIN Focalisation sur les aspects financiers

FOCHUM Focalisation sur les aspects humains

FOCTEC Focalisation sur les aspects techniques

FOCONC Focalisation sur la position concurrentielle du partenaire 
TABLEAU 5

Gestion de l'alliance (suite 1)

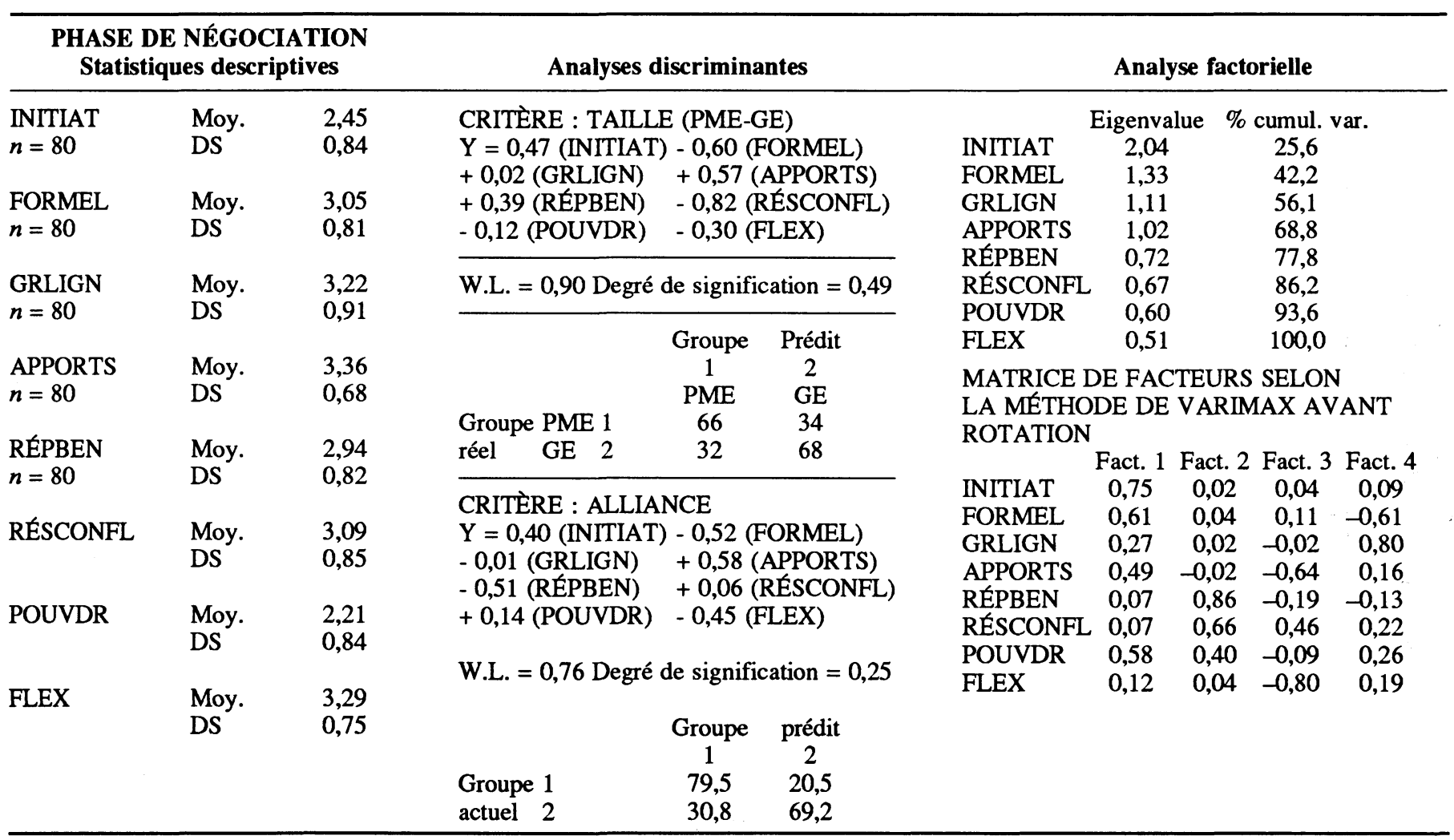


GraphiQue du TABLEAU 5

Diagramme des variables dans l'espace des facteurs 1 et 2

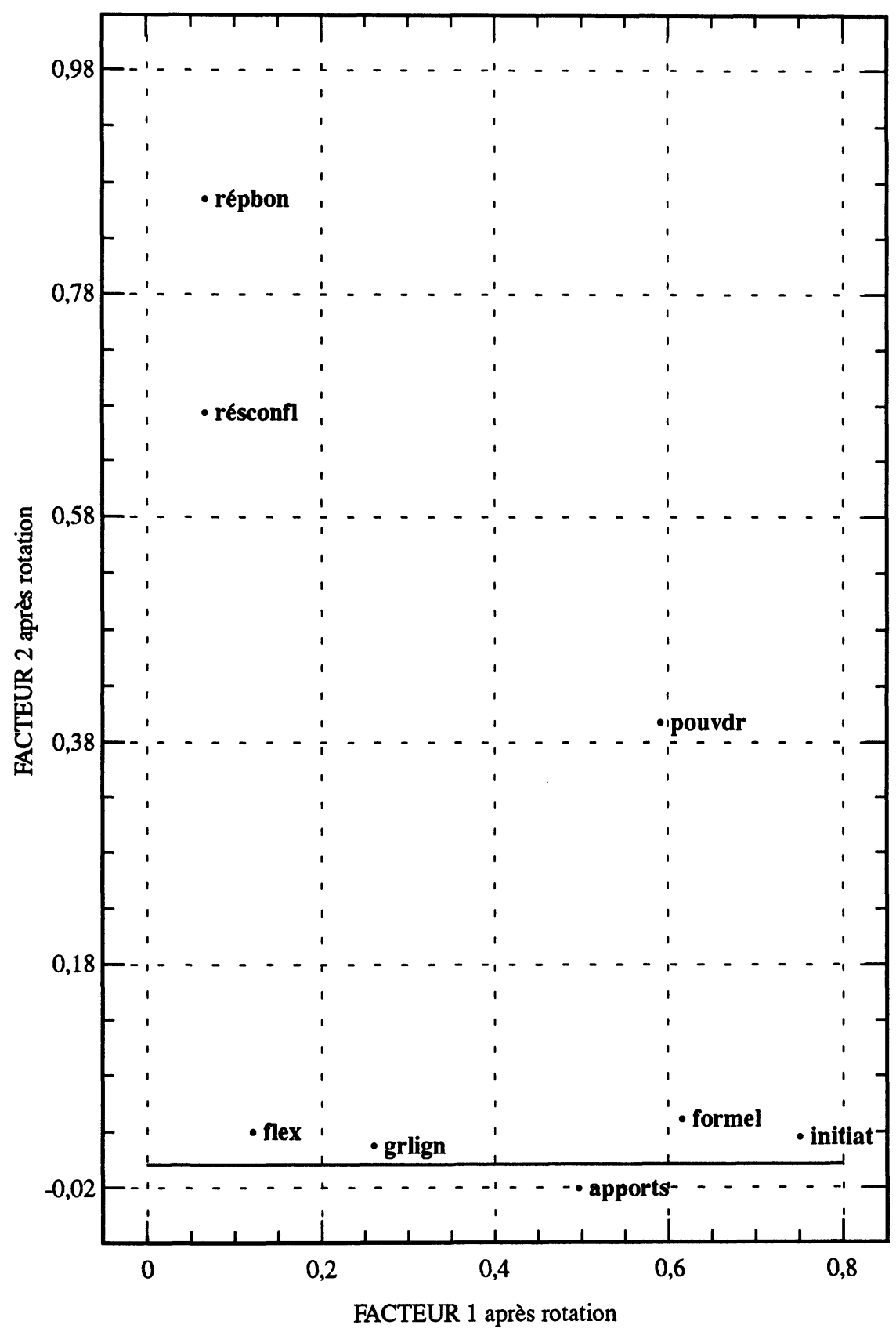

Revue internationale P.M.E., vol. 4, $n^{\circ}$ 2, 1991 


\section{LISTE DES VARIABLES}

\section{TABLEAU 5}

Gestion de l'alliance - phase de négociation

\begin{tabular}{ll}
\hline INITIA & Avoir l'initiative de la négociation \\
FORMEL & Existence d'un contrat formel et détaillé \\
APPORTS & Fixation précise des apports de chaque partie \\
GRLIGN & Fixer les grandes lignes de l'accord \\
RÉPBEN & Prévoir la répartition des bénéfices \\
RESCONFL & Prévoir les modes de résolution des conflits \\
POUVDR & Avoir le pouvoir de droit \\
FLEX & Être flexible
\end{tabular}


TABLEAU 6

Gestion de l'alliance

Phase de mise en œuvre

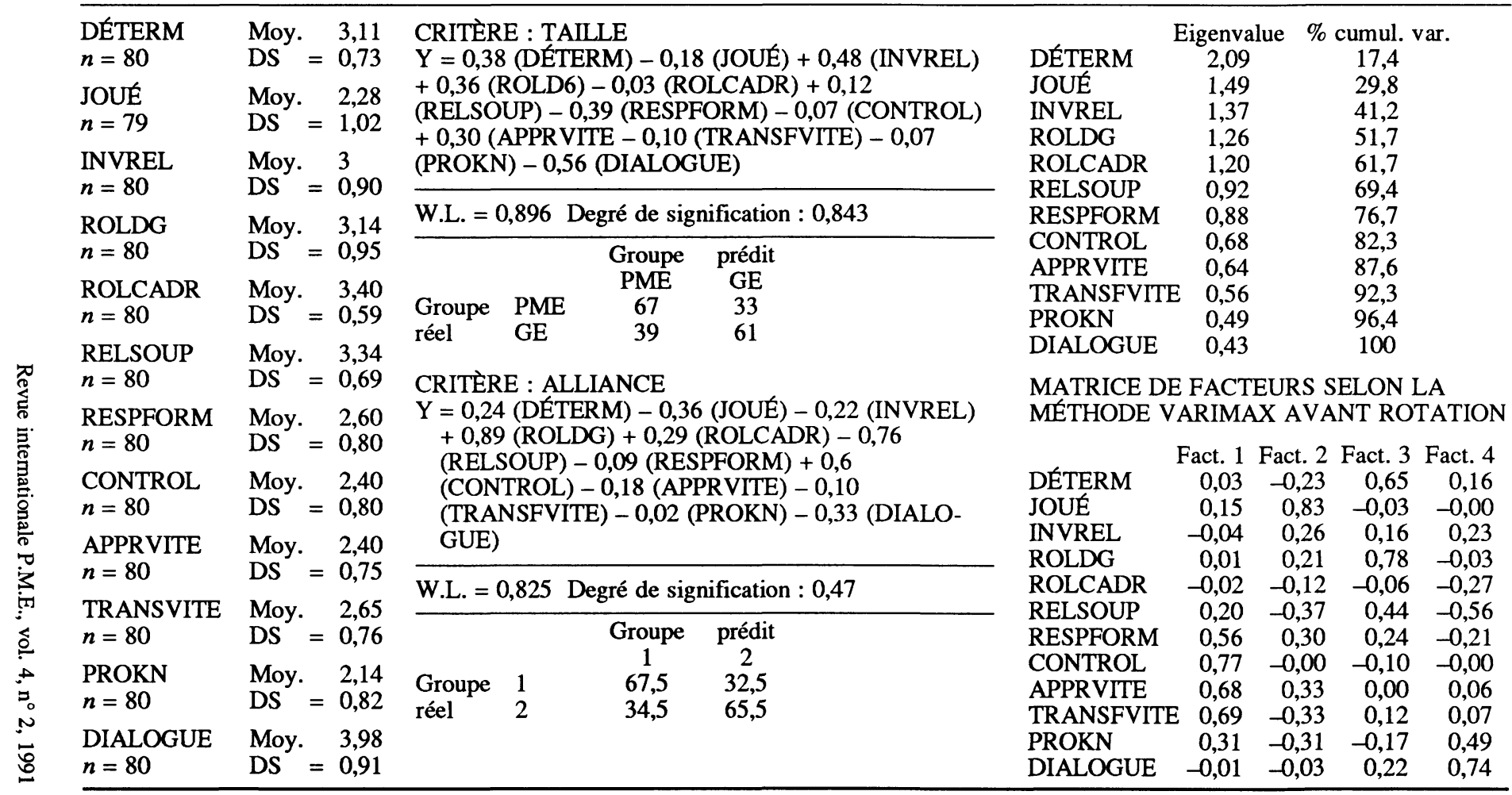


GRAPHQUE DU TABLEAU 6

Diagramme des variables dans l'espace des facteurs 1 et 2

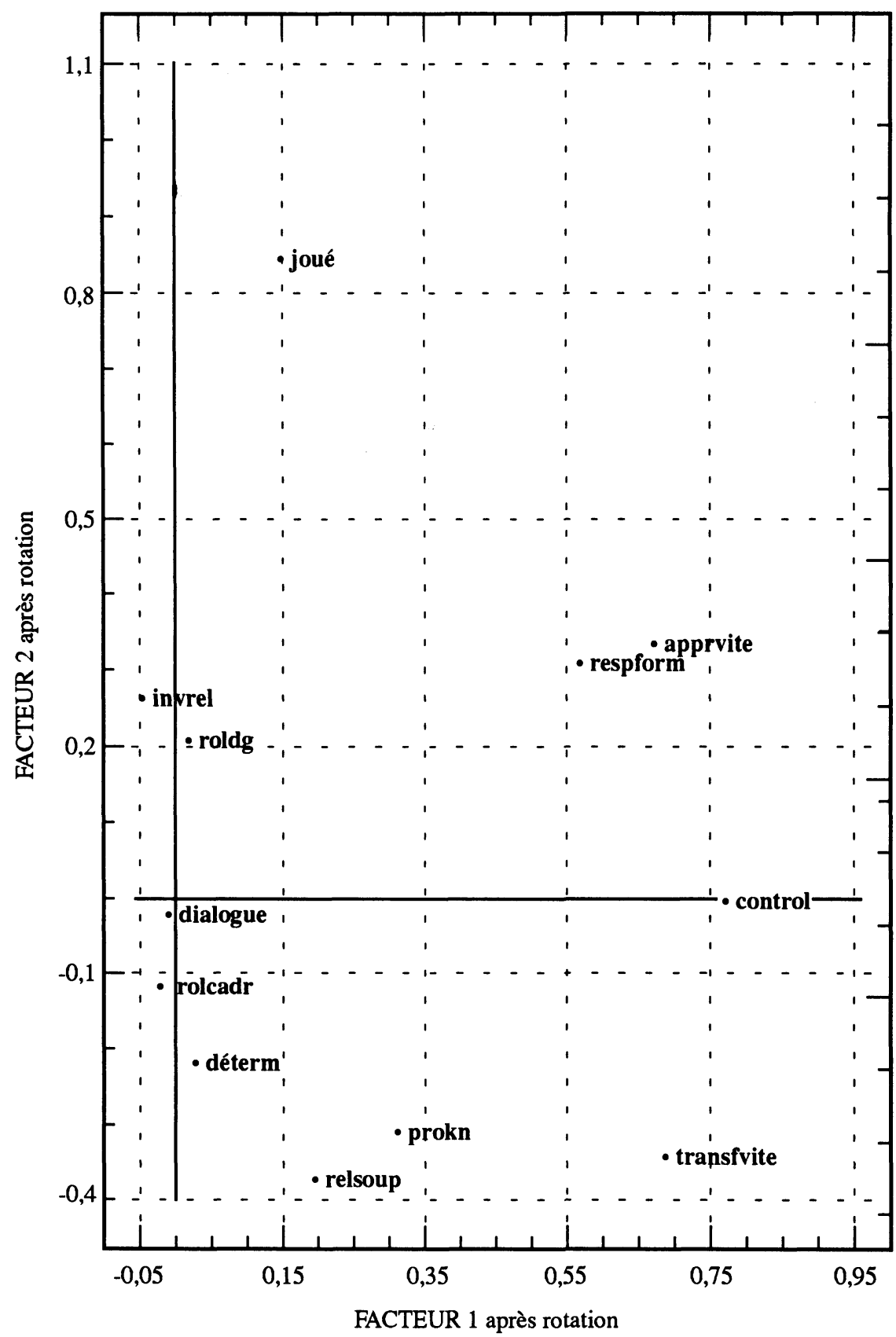

Revue internationale P.M.E., vol. 4, $\mathrm{n}^{\circ}$ 2, 1991 


\section{LISTE DES VARIABLES}

\section{TABLEAU 6 \\ Gestion de l'alliance - phase de mise en oeuvre}

\begin{tabular}{|c|c|}
\hline DÉTERM & Ce stade est déterminant \\
\hline JOUÉ & À ce stade tout est joué \\
\hline INVREL & À ce stade, il faut investir dans la relation \\
\hline ROLDG & Le rôle de la direction générale est essentiel \\
\hline ROLCADR & Ce sont les cadres qui jouent le rôle essentiel \\
\hline RELSOUP & Il faut être souple dans les relations avec les partenaires \\
\hline RESPFORM & Il faut respecter l'accord formel \\
\hline CONTROL & Il faut contrôler l'autre partie \\
\hline APPRVITE & Ce qui compte, c'est d'apprendre vite du partenaire \\
\hline TRANSFVITE & $\begin{array}{l}\text { Ce qui compte, c'est de transférer vite cet apprentissage à l'ensemble } \\
\text { de l'organisation }\end{array}$ \\
\hline PROKN & Ce qui compte, c'est de protéger notre savoir-faire \\
\hline DIALOGUE & Ce qui compte, c'est de dialoguer régulièrement avec le partenaire \\
\hline
\end{tabular}




\section{Annexe bibliographique 1}

CHESNAIS (1988), « Les accords de coopération techniques entre firmes indépendantes », STI (Sciences, Technologies, Industries), $n^{\circ} 4$, décembre, p. 51-128.

JACQUEMIN, A. et B. REMICHE (édit.) (1988), Coopération entre entreprises, entreprises conjointes, stratégies industrielles et pouvoirs publics, Bruxelles, De Boeck.

Petrella, R. (1987), Conference transcript EEC, Brussels. 